\title{
COMUNICACIÓN
}

\section{Parásitos de la liebre (Lepus europaeus Pallas, 1778): estudio en dos zonas geográficas de Chile}

\author{
DANIEL GONZÁLEZ-ACUÑA*, PAULA REBOLLEDO*, OSCAR SKEWES*, \\ LUCILA MORENO* y DOLORES CASTRO**
}

\section{HARE PARASITES (Lepus europaeus) IN TWO GEOGRAPHICAL ZONE OF CHILE}

The parasites of 40 Lepus europaeus (hares) were analyzed (20 of the Nuble Province and 20 of Ultima Esperanza) caught between January 2003 and August 2004. In the Nuble province three endoparasites species were isolated: Trichostrongylus retortaeformis (60\%), Passalurus ambiguus (5\%) and Eimeria spp. (45\%). In Ultima Esperanza only T. retortaeformis was isolated with $60 \%$ of infection. The ectoparasite species isolated corresponded to the Phthiraptera Haemodipsus lyriocephalus in a hare of the Nuble Province. No significant differences in the presence of gastrointestinal parasites were found with respect to sex and age of the hare. Nor was there any significant difference between parasitism of $\boldsymbol{T}$. retortaeformis in the two studied zones.

Key words: Lepus europaeus, Survey, Wild Animal Parasites, Chile.

\section{INTRODUCCIÓN}

La liebre, Lepus europaeus fue introducida en Argentina desde Europa por el cónsul alemán hacia el año $1880^{1}$. Su llegada a Chile aún está en discusión, mientras algunos autores sostienen que se dispersó hacia el país a través de los Andes, colonizando gran parte del territorio nacional ${ }^{1}, \mathrm{y}$ un autor afirma que la liebre fue introducida por primera vez en Chile desde Alemania, en 1896, en la zona de Última Esperanza y luego introducida en las provincias de Osorno y Valdivia en el siglo $\mathrm{XX}^{2}$. Su distribución comprende desde el límite norte del Río Copiapó y, desde ésta área, cubre prácticamente todo territorio hasta el extremo sur de Chile, con excepción de las islas. Es abundante en la provincia de Magallanes y Aysén, pero no en las inmediaciones del Estrecho de Magallanes ${ }^{3}$.
La liebre al igual que el conejo silvestre, puede constituir una amenaza debido a la capacidad de actuar como portador, huésped intermediario y reservorio de infecciones de importancia para animales domésticos y el hombre ${ }^{4,5}$. Estudios realizados en otros países han podido constatar que la liebre es hospedador de numerosos endoparásitos incluyendo Trematodos, Cestodos, Nematodos y distintas especies de coccidias 6 .

En relación a ectoparásitos, se ha registrado una especie de Phthiraptera correspondiente a Haemodipsus lyriocephalus en liebres de Eurasia, Nueva Zelanda y Argentina ${ }^{7-9}$, además, los ixodidos Haemophysalis leporipalustris, $H$. longicornis, Hyaloma marginatum e Ixodes ricinus en liebres de Europa, Nueva Zelanda y Argentina ${ }^{10-12}$, los ácaros Cheyletiella parasitivorax y Listrophorus gibbus en Europa ${ }^{7}$ y un

\footnotetext{
Facultad de Medicina Veterinaria, Universidad de Concepción, Casilla 537, Chillán, Chile.

** Facultad de Ciencias Naturales y Museo de la Plata, Paseo del Bosque s/n, 1900, La Plata, Argentina.
} 
Siphonaptera Ctenocephalides canis en Nueva Zelanda ${ }^{11}$.

El presente estudio tiene como objetivo determinar la fauna parasitaria de la liebre en dos zonas geográficas de Chile, primer estudio realizado en la mencionada especie en nuestro país.

\section{MATERIAL Y MÉTODO}

Entre enero del 2003 y agosto del 2004, durante la temporada de caza (Ley $\mathrm{N}^{\circ} 19.473$ ), se obtuvieron 20 liebres en la Provincia de Ñuble (VIII Región) (36 41'-36 42' S 72 $16^{\circ}-72^{\circ} 12^{\prime}$ W) y 20 liebres en Última Esperanza (XII Región) $\left(48^{\circ} 36^{\prime}-56^{\circ} 30^{\prime} \mathrm{S} 66^{\circ} 25^{\prime}-75^{\circ} 40^{\prime} \mathrm{W}\right)$. A cada ejemplar se le determinó el sexo, peso y tamaño. Posteriormente, fueron almacenados en forma individual en bolsas plásticas herméticas para así no perder ectoparásitos y más tarde ser procesados en el laboratorio.

Los Phthiraptera fueron colectados en forma manual desde cada ejemplar el mismo día de su captura, se conservaron en alcohol $70 \%$ y posteriormente fueron limpiados en $\mathrm{KOH}$ al $20 \%$, llevados por soluciones ascendentes de alcohol (40\%, $80 \%$ y $100 \%)$, aclarados durante 24 horas en aceite de clavo, para finalmente ser montados en Bálsamo de Canadá ${ }^{13}$. El material estudiado fue depositado en la colección del laboratorio de Zoología de la Facultad de Medicina Veterinaria de la Universidad de Concepción.

Las técnicas de colecta, fijación y tinción de los endoparásitos se basaron en una metodología descrita previamente ${ }^{14}$. De las porciones de recto de cada ejemplar se obtuvo una muestra de material fecal, la cual fue analizada mediante el método de flotación tradicional ${ }^{15}$.

A partir de los endoparásitos aislados se calculó la intensidad media, el rango y la abundancia ${ }^{16-17}$. Se utilizó la prueba estadística de $t$ de Student a fin de determinar si existe relación entre el sexo de las liebres y el nivel de infección de parásitos gastrointestinales encontrados y además para determinar si existían mayor proporción de endoparásitos hembras o machos en las liebres capturadas. La relación hembras/machos se obtuvo dividiendo el total de parásitos hembras por el total de parásitos machos, según el tipo de especie parasitaria.

\section{RESULTADOS Y DISCUSIÓN}

El 60,2\% (25/40) de las liebres examinadas resultaron positivas a la presencia de nematodos gastrointestinales, cifra que es bastante menor a la que se ha encontrado en otros estudios similares realizados en otros lugares del mundo. Es así, como en estudios realizados en Alemania ${ }^{18}$ y en Austria ${ }^{7}$, se obtuvo un $96,7 \%$ y $90,0 \%$ de de infección a parásitos gastrointestinales respectivamente, siendo en su mayoría infecciones por Trichostrongylus retortaeformis, uno de los parásitos más comunes dentro de los lago-morfos ${ }^{19,21}$.

El parásito que se encontró con una mayor prevalencia en nuestro estudio $(60 \%)$ fue el $T$. retortaeformis (24/40\%). El Passalurus ambiguus se encontró tan sólo en una liebre $(1 / 20)$ proveniente de la Provincia de Nuble (5\%), cifra que es ligeramente mayor que la obtenida en Alemania ${ }^{18} \mathrm{y}$ Austria $^{7}$, en donde se encontró en un 3,4\% y 1,2\% respectivamente. En ninguna de las 20 liebres de Última Esperanza se encontró este parásito.

Tabla 1. Prevalencia de infección, carga parasitaria y relación hembras/machos de Trichostrongylus retortaeformis colectados en liebres (Lepus europaeus), capturadas en la Provincia de Ñuble y Útima Esperanza

\begin{tabular}{|c|c|c|c|c|c|c|c|c|c|c|c|}
\hline Sector & $\begin{array}{c}\text { Liebres } \\
\text { exami- } \\
\text { nadasa } \\
\mathbf{N}^{\circ}\end{array}$ & $\mathbf{N}^{\mathbf{o}}$ & $\begin{array}{c}\text { Positivas } \\
\text { T. retorta- } \\
\text { eformis } \\
\%\end{array}$ & $\mathbf{N}^{o}$ & $\begin{array}{c}\text { Ejemplares } \\
\text { T. retorta- } \\
\text { eformis } \\
\text { Rango }\end{array}$ & $\begin{array}{l}\text { ntensidad } \\
\text { media }\end{array}$ & A & H & $\mathbf{M}$ & $\mathbf{L}$ & $\mathrm{RH} / \mathrm{M}$ \\
\hline $\begin{array}{l}\text { Provincia } \\
\text { de Ñuble }\end{array}$ & 20 & 12 & 60 & 2.617 & $1-1.463$ & 218,08 & 64,42 & 1.196 & 1.043 & 378 & $1,1 / 1$ \\
\hline $\begin{array}{l}\text { Última } \\
\text { Esperanza }\end{array}$ & 20 & 12 & 60 & 423 & $10-82$ & 35,25 & 10,57 & 187 & 194 & 42 & $0,9 / 1$ \\
\hline Total & 40 & 24 & 60 & 3.040 & & & & 1.383 & 1.237 & 420 & $1 / 1$ \\
\hline
\end{tabular}

$\mathrm{H}=$ Hembra, $\mathrm{RH} / \mathrm{M}=$ Relación hembra/macho, $\mathrm{M}=$ Macho, $\mathrm{A}=$ Abundancia, $\mathrm{L}=$ Larva. 
Con respecto a estos parásitos en otros lagomorfos del país, se puede indicar que en estudios realizados en conejos silvestres de Chile $^{22-23}$, se han obtenido prevalencias de infección por nematodos gastrointestinales que oscilan entre el $89,3 \%$ y el $99,1 \%{ }^{22-24}$. En todos esos estudios el nematodo que se encontró con más frecuencia fue el $T$. retortaeformis, y el $P$. ambiguus se encontró presente en el $57,6 \%$, $67,5 \%{ }^{23}$ y $87,1 \%{ }^{24}$ de los conejos examinados, cifras bastante mayores a las obtenidas en el presente estudio.

Los ejemplares adultos de $T$. retortaeformis colectados en Nuble (1.196 hembras y 1.043 machos) y Última Esperanza (187 hembras y 194 machos) correspondieron a $85,5 \%$ y $90,0 \%$ respectivamente de los ejemplares de la especie recolectados (Tabla 1), esto podría indicar que las poblaciones de $T$. retortaeformis no están creciendo marcadamente, y las reinfecciones con el parásito son bajas. En Alemania, en $1996^{25}$ se encontró un $49 \%$ en un estudio realizado en 94 liebres, el promedio fue de 102 vermes/liebre, datos que se acercan a los obtenidos en Nuble (218,08 vermes/liebre). Por otro lado, en el estudio realizado en conejo silvestre realizado en la comuna de Florida ${ }^{23}$, muestra que la carga parasitaria de éste nematodo varió entre 13 y 9.375 vermes, con un promedio de 1.292 vermes/ conejo, superando con creces los resultados obtenidos en el estudio en Alemania ${ }^{25}$ y los del presente estudio. Otro estudio realizado en Europa $^{26}$, registró para $T$. retortaeformis de 0 a 2.876 vermes, resultados que se acercan a los obtenidos en Nuble, pero no a los de Última Esperanza, los cuales fueron más bajos (Tabla 1).

Si comparamos los resultados obtenidos en el presente estudio con los obtenidos en Europa ${ }^{26}$ que registran para $P$. ambiguus infecciones que van desde 0 a 19.373, se puede concluir que el parasitismo en la liebre para Nuble (5\%) es baja y nula para Última Esperanza. Los resultados obtenidos indicarían que $P$. ambiguus se adaptaría en Chile mejor en conejos que en liebres, sin embargo, para asegurar esta hipótesis habría que realizar estudios en un mayor número de ejemplares y por periodos más prolongados.

Según los resultados, la relación hembras/ macho de $T$. retortaeformis, para Ñuble fue superior a lo obtenido para Última Esperanza (Tabla 1), Forstner \& $\operatorname{Ilg}^{18}$ obtuvieron 1,4/1 $( \pm 1)$ para los diferentes distritos en estudio, datos muy similares a los registrados en Ñuble. Estos resultados indican que no existen diferencias significativas entre las poblaciones de hembras y machos, por lo que seguramente el periodo de vida de ambos parásitos sería similar.

Entre el porcentaje de infección por $T$. retortaeformis en liebres adultas de Ñuble $(66,6 \%)$ y Última Esperanza $(73,3 \%)$ no hubo diferencias estadísticas significativas $(\mathrm{p}>0,05)$. En las liebres juveniles el porcentaje fue de $40 \%$ y $20 \%$ para Nuble y Última Esperanza respectivamente, diferencia que tampoco fue significativa $(\mathrm{p}>$ $0,05)$. Estas similitudes hacen suponer que las diferencias que pudiese haber entre ambos ambientes Ñuble y Última Esperanza no influirían sobre el ciclo biológico del T. retortaeformis.

El nivel de infección por $T$. retortaeformis por sexo (Tabla 1) no muestra diferencias estadísticas además al comparar el sexo y la edad de las liebres con la presencia de parásitos, se determinó que no existe una relación entre estos parámetros.

La coccidiosis es una de las endoparasitosis más frecuente de las liebres ${ }^{26}$. La incidencia reportada en otros estudios va desde un $66 \%$ a $94 \%{ }^{18,26,27}$. En el presente estudio sólo en nueve liebres de Ñuble (45\%), hubo presencia de Eimeria $\mathrm{sp}$.

No se observó presencia de Fasciola hepatica en las liebres de ambas zonas estudiadas. A pesar de las bajas prevalencias halladas en Argentina ${ }^{28}$, el rol de L. europaeus en la transmisión del parásito podría ser importante si se considera que la especie comparte ambientes con el ganado y el caracol.

En relación a ectoparásitos sólo se logró colectar diez especimenes del Phthiraptera Haemodipsus lyriocephalus en una liebre proveniente de la Provincia de Ñuble. Haemodipsus tiene 6 especies, asociadas a Lagomorpha Leporidae $^{9}$, este Phthiraptera ha sido descrito en Europa en L. timidis, L. glacialis y L. europaeus ${ }^{29}$, también ha sido descrito en Nueva Zelanda y Argentina. Con el presente estudio se cita a $H$. lyriocephalus por primera vez en Chile.

\section{RESUMEN}

Se analizaron los parásitos de 40 liebres (Lepus europeaus) (20 de la Provincia de Ñuble y 20 de Última Esperanza) capturadas entre enero de 2003 y agosto de 2004. En la Provincia de Nuble se aislaron tres especies de endoparásitos: 
Parásitos de Lepus europaeus en dos zonas geográficas de Chile - D. González et al.

Trichostrongylus retortaeformis (60\%), Passalurus ambiguus (5\%) y Eimeria spp. (45\%). En Última Esperanza se encontró sólo la especie $T$. retortaeformis $(60 \%)$. Se aisló además una especie de ectoparásito correspondiente al Phthiraptera Haemodipsus lyriocephalus en una liebre de la Provincia de Ñuble.

No se estableció ninguna relación significativa entre la presencia de los parásitos gastrointestinales con el sexo y edad de las liebres. Tampoco hubo una diferencia significativa entre el parasitismo provocado por $T$. retortaeformis en las dos zonas estudiadas.

\section{REFERENCIAS}

1.- HOUSSE P R. Animales salvajes de Chile, en su clasificación moderna: su vida y sus costumbres. Ediciones de la Universidad de Chile. Santiago, Chile. 1953.

2.- CAMPOS C H. Mamíferos Terrestres de Chile: Guía de reconocimiento. Vol. 5. Marisa Cúneo Ediciones. Valdivia, Chile. 1996.

3.- GRIGERA D E, RAPOPORT E H. Status and distribution of the European Hare in South America. J Mamm 1983; 64: 163-6.

4.- CABELLO C. Consideraciones sobre la importancia de las enfermedades de los animales silvestres. Rev Soc Med Vet Chile 1974; 24: 24-8.

5.- COURTIN S, ALCAÍNO H, PLAZA J, FERRIERE G. Platelmintos del conejo silvestre (Oryctolagus cuniculus) en la cordillera de Nahuelbuta. Chile. Arch Med Vet 1979; 11: 23-6.

6.- BOWMAN D D, LYNN R C, EBERHARD M L. Parasitología para veterinarios. $8^{\mathrm{a}}$ Edición. Elsevier España, S.A. 2004.

7.- KUTZER E, FREY H. Die Parasiten der Feldhasen (Lepus eurapaeus) in Österreich. Berl Münch Tierärztl Wschr 1976; 89: 480-3.

8.- DURDEN L A, MUSSER G G. The sucking lice (Insecta, Anoplura) of the world: a taxonomic checklist with records of mammalian hosts and geographic distributions. Bull Amer Mus Natur Hist 1994; 218: 1-90.

9.- CASTRO D DEL C, CICCHINO A C. Anoplura. pp 125-139. En Morrone J J Coscaron S (Ed.). Biodiversidad de artrópodos Argentinos. Ediciones Sur. La Plata, Argentina. 1998.

10.- KÖTSCHE W, GOTTSCHALK C. Krankheiten der Kaninchen und Hasen. 4 Auflage. Gustav Fischer Verlag Jena. German, 1990. 359 p.

11.- TENQUIST J D, CHARLESTON W A G. A revision of the annotated checklist of ectoparasites of terrestrial mammals in New Zeland. J Royal Society New Zel 2001; 31: 481-542.

12.- GUGLIELMONE A A, ESTRADA-PEÑA A, KEIRANS J E, ROBBINS R G. Ticks (Acari: Ixodidae) of the Neotropical zoogeographic region. Int Cons Ticks Tick-Borne Dis, Atalanta, Houten, The Netherlands, 2003. 174 p.

13.- PALMA R. Slide-mounting of Lice: a Detailed Description of the Canada Balsam technique. New Zel
Entomol 1978; 6: 432-6.

14.- KINSELLA J M, FORRESTER D J. Helminth parasites of the Florida duck, Anas platyrhynchos fulvigula. Proc Helminthol Soc Wash 1972; 39: 173-6.

15.- BOCH J, SUPPERER R. Veterinärmediznische Parasitologie (4a Ed.). Verlag Paul Parey. Berlin y Hamburgo. Alemania. 1992.

16.- MARGOLIS L, ESCH G W, HOLMES J C, KURIS A M, SHAD G A. The use of ecological termsin parasitology (report of an ad hoc committee of the American Society of Parasitologist). J Parasitol 1982; 68: 131-3.

17.- BUSH A, LAFFERTY K, LOTZ J, SHOSTACK A. Parasitology meets ecology on its own terms: Margolis et al. revisited. J Parasitol 1997; 83: 575-83.

18.- FORSTNER M J, ILG B. Untersuchungen über die Endoparasiten des Feldhasen (Lepus europaeus) und Versuche zu ihrer Bekämpfung. Z Jagdwiss 1982; 28: 169-77.

19.- BOAG B. Helminth parasites of the wild rabbit Oryctolagus cuniculus (L.) in north east England. Helminthol Abst 1972; 42: 64.

20.- GAIARDI S. Gastro-intestinal nematodes of rabbits in the Province of Bologna. Helminthol Abst 1977; 46: 462.

21.- DUNSMORE J M R. Parasitism in wild rabbits. Helminthol Abst 1979; 48: 1215.

22.- CATTÁN P, TAGLE L. Estudio preliminar de la helmintiasis gastrointestinal en el conejo silvestre. Oryctolagus cuniculus. Rev Soc Med Vet Chile 1974; 24: 34-42.

23.- ALCAINO H, HERRERA M, GORMAN T. Helmintiasis e infección por Toxoplasma gondii en conejos silvestres (Oryctolagus cuniculus)de la zona precordillerana del Rio Renaico, Chile. Parasitol al Día 1990; 14: 86-90.

24.- MERELLO E T. Determinación del endoparasitismo en conejos silvestres (Oryctolagus cunniculi) capturados en la comuna de Florida. Concepción. Memoria de título Med. Vet. Universidad de Concepción, Fac Med Vet, Área de Ciencias Agropecuarias. Chillán, Chile. 1980.

25.- BÜTTNER S. Gesundheitszustand von Feldhasen (Lepus europaeus) in der Schweiz. Aus dem Institut für Tierpathologie der Universität Bern. Alemania. 1996.

26.- ALLGÖWER R. Der Parasitenbefall von Feldhasen aus der Oberrheinebene und seine intraspezifische Bedeutung. Z Jagdwiss 1992; 38: 116-27.

27.- BARTH D, BRÜLL U. Magen-Darmhelminthen und Kokzidien beim Feldhasen (Lepus europaeus Pallas) und ihre Bekämpfung mit Thibenzole und Theracanzan. Z Jagdwiss 1975; 21: 15-34.

28.- KLEIMAN F, GONZÁLEZ N, RUBEL D, WISNIVEKSY C. Fasciola hepatica (Linnaeus, 1758) (Trematoda, Digenea) en liebres europeas (Lepus europaeus, Pallas 1778) (Lagomorpha, Leporidae) en la región Cordillerana Patagónica, Chubut, Argentina. Parasitol Latinoam 2004; 59: 68-71.

29.- FERRIS G F. The sucking lice. Mem Pacific Coast Entomol Society Volumen 1. 1951.

Agradecimientos: al Servicio Agrícola y Ganadero (XII Región), quien bajo el proyecto Estudio de Relaciones Forrajeras de los principales Herbívoros Silvestres con la Ganadería de la XII Región, financio parte del presente estudio. Además se agradece a Jorge Solís quien realizo las correspondientes pruebas de laboratorio. 